[Article In Press at Aging, Neuropsychology and Cognition]

\title{
Neuromodulation as a cognitive enhancement strategy in healthy older adults: promises and pitfalls
}

Running title: Neuromodulation in healthy older adults

Ana R. S. Martins ${ }^{1) 2)}$ (anamartins@ @pce.uc.pt), Felipe Fregni ${ }^{3)}$ 4) (felipe.fregni@ppcr.org), Marcel Simis ${ }^{5)}$ (marcelsimis@gmail.com) and Jorge Almeida ${ }^{1)}{ }^{2)}$ (jorgealmeida@fpce.uc.pt)

${ }^{1)}$ Faculty of Psychology and Educational Sciences, University of Coimbra, Rua do Colégio Novo, 3001-802 Coimbra, Portugal; ${ }^{2)}$ Proaction Laboratory, Faculty of Psychology and Educational Sciences, University of Coimbra, Rua do Colégio Novo, 3001-802 Coimbra, Portugal; ${ }^{3)}$ Spaulding Neuromodulation Center, Department of Physical Medicine \& Rehabilitation, Spaulding Rehabilitation Hospital and Massachusetts General Hospital, Charlestown, MA 02129, USA; ${ }^{4)}$ Harvard Medical School, Boston, MA 02115, USA; ${ }^{5}$ Service of Interdisciplinary Neuromodulation, Department and Institute of Psychiatry, University of São Paulo, São Paulo, Brazil and Division of Neurology, Santa Casa Medical School, Sao Paulo, Brazil.

Corresponding author: Jorge Almeida, Faculty of Psychology and Educational Sciences, University of Coimbra, Rua do Colégio Novo, 3001-802 COIMBRA, Phone: 00351239247418 , Fax: 00351 239851465, Email: jorgealmeida@fpce.uc.pt

Funding Statement/Acknowledgments: This research was funded by a grant from the Bial Foundation (Grant 2014/495). This research was funded also by a grant from the Portuguese Foundation for Science and Technology (FCT; UID/PSI/00730/2013), under FEDER, COMPETE, and FCT funds to the Cognitive and Behavioural Center for Research and Intervention, FPCE-UC. A. M. was supported by an FCT grant (UID/PSI/00730/2013). 
Authors Disclosures: No conflict of interest has been declared by the authors.

\title{
Neuromodulation as a cognitive enhancement strategy in healthy older adults: promises and pitfalls
}

\begin{abstract}
Increases in life expectancy have been followed by an upsurge of age-associated cognitive decline. Transcranial magnetic stimulation (TMS) and transcranial direct current stimulation (tDCS) have risen as promising approaches to prevent or delay such cognitive decline. However, consensus has not yet been reached about their efficacy in improving cognitive functioning in healthy older adults. Here we review the effects of TMS and tDCS on cognitive abilities in healthy older adults. Despite considerable variability in the targeted cognitive domains, design features and outcomes, the results generally show an enhancement or uniform benefit across studies. Most studies employed tDCS, suggesting that this technique is particularly well-suited for cognitive enhancement. Further work is required to determine the viability of these techniques as tools for long-term cognitive improvement. Importantly, the combination of TMS/tDCS with other cognitive enhancement strategies may be a promising strategy to alleviate the cognitive decline associated with the healthy aging process.
\end{abstract}

Keywords: neuromodulation; healthy; cognitive function; TMS; tDCS 
Word count $=$ about 9.000 words

\section{Introduction}

Life expectancy has been steadily increasing in the developed world (Mathers et al. 2014). It is expected that by 2020 , one billion people - i.e., more than $20 \%$ of the world population - will be older than 60 years (Imhof et al. 2007). This brings important challenges to individuals and society as a whole. Perhaps one of the central issues is related with an increase in the incidence of age-associated cognitive decline (Bishop et al. 2010). As people grow older, the structural and functional changes that occur in their brain lead to an overall deterioration of cognitive functioning (Glisky 2007), potentially compromising quality of life and social interaction. How these structural and functional changes come to be, and which factors may be driving forces in promoting cognitive decline in healthy older adults is still under debate. For instance, structural and cognitive decline may be highly dependent on amyloid-B levels (Lim et al. 2013), or individual differences and quality of life/socio-economics status (Friedman 2013). Nevertheless, it is undisputed that healthy older adults show anatomical and physiological brain modifications that interfere with cognitive performance in a myriad.

There are currently several methods available to assess cognitive functioning and, specifically, age-related decay in cognitive performance. However, defining the limits of normal and abnormal performance may still be a challenge. According to the Fifth Edition of the Diagnostic and Statistical Manual of Mental Disorders (DSM-5; American Psychiatric Association 2013), an indication of modest cognitive decline from a previous level of performance in one or more domains is a criterion for agingassociated abnormal performance. Concomitantly, neuropsychological testing also allows for assessing cognitive decline and describing its severity. However, determining whether observed cognitive decline is a byproduct of the normal aging process, or 
whether it is due to more severe insults to an individual's brain (e.g., mild cognitive impairment) is still problematic.

In recent years, there has been an upsurge of interest in exploring nonpharmacological strategies to maintain or enhance cognitive functioning in healthy older adults, to prevent or delay such functional disabilities and diminish the impact of this decline in their functional capacity and quality of life. Previous studies have shown that single interventions such as training of particular strategies (e.g., mnemonic strategies), multimodal approaches (e.g., lifestyle changes, physical exercise; Lustig et al. 2009), and the combination of different types of interventions (Kraft, 2012, Bamidis et al. 2014), can lead to cognitive enhancement in healthy older adults. Nonetheless, given the heterogeneity of studies it is still not clear what the benefits and impact of these interventions are (Angevaren et al. 2008 and Lampit et al. 2014).

One family of techniques that has been showing promising results in maintaining or improving cognitive performance in healthy (Zimerman and Hummel 2010) and clinical populations (Demirtas-Tatlidede et al. 2013) is non-invasive brain stimulation (NIBS). These techniques rely on the potential to transiently influence behavior by facilitating or inhibiting neural activity. Over the years, different techniques have been explored, showing favorable motor and cognitive behavioral effects (Hummel and Cohen 2006a and Reis et al. 2009). To date, Transcranial Magnetic Stimulation (TMS) and transcranial Direct Current Stimulation (tDCS) are among the most commonly used approaches (Shafi et al. 2012). However, consensus has not yet been reached as to the efficacy of these techniques in improving cognitive functioning in healthy older adults (Zimerman and Hummel 2010). Here, we will systematically review the literature to clarify the role of NIBS techniques, specifically TMS and tDCS, in improving cognitive functioning in healthy older adults. We will assess and discuss the available data, and 
provide considerations and recommendations regarding these techniques. Our goal is then to acknowledge the promises and recognize potential pitfalls of these techniques in this field.

\section{Age related cognitive changes}

To distinguish between normal and pathological brain aging is not a straightforward process, mostly because changes are not homogeneous or uniform across individuals. It is clear that aging is associated with brain modifications that may affect cognition and limit functional capacity (Glisky 2007), but the processes involved in this structural and functional decline, as well as potential interventions to slow these changes are less clear. Research efforts have been carried out for decades to understand the epidemiological, clinical, morphological, genetic and molecular aspects of ageassociated cognitive changes. As a human brain ages, neuronal loss occurs and the brain tends to shrink in volume (Park and Reuter-Lorenz 2009). This age-associated cortical shrinkage has been linked to cognitive deficits in various domains. Decline in gray and white matter structures in the brain also increases with age (Peters 2006), compromising neural integrity that is critical for cognitive performance, as shown by recent structural and functional neuroimaging studies (Salat 2011 and Grady 2012). Changes in the levels of different neurotransmitters also impact on age-related cognitive functioning. Specifically, reductions in the levels of dopamine, serotonin and acetylcholine are particularly pronounced, compromising a wide range of cognitive domains, including attention, working memory and inhibition (Braver and Barch 2002 and Mattson et al. 2004).

These changes are not homogenously distributed across different brain regions, nor are they consistent between different individuals. Importantly, the frontal lobes 
(Peters 2006) and the hippocampus (Den Heijer et al. 2010) are often described as the primarily affected regions. In agreement, the most widely experienced age-associated cognitive changes are memory decline, associated with hippocampal integrity, and executive function, linked to frontal lobe function (Mungas et al. 2005 and Kramer et al. 2007).

Several interventions have been proposed and implemented to slow or reverse these and other cognitive changes (Lustig et al. 2009), but it is still unclear how we can successfully promote healthy aging and prevent or slow down age-associated cognitive decline. With the advent of NIBS, several studies have examined the effects of different techniques on cortical excitability and behavior. Such experiments have contributed to a better understanding of the mechanisms underlying cognitive processing. However, most of these studies have been focusing on younger adults or patients with neurological diseases (Nitsche and Paulus 2009 and Hartwigsen 2014). Specifically, one of the fields that has recently gained more attention regarding cognitive improvement following the use of neuromodulation techniques is the field of neurodegenerative dementias. A recent systematic review (Elder and Taylor, 2014) explored the use of TMS and tDCS in the major dementias, including Alzheimer's disease, vascular dementia, dementia with Lewy bodies, frontotemporal dementia, Parkinson's disease with dementia as well as the potential pre-dementia stages of mild cognitive impairment and Parkinson's disease. Most of the reviewed studies were conducted in patients with Alzheimer's disease (13 studies) and mild cognitive impairment (4 studies).

Neuromodulation techniques were used in one study focusing on dementia with Lewy bodies. Fourteen studies were found that focused on Parkinson's disease, 12 of which showed cognitive or neuropsychiatric improvement. From a total of 18 studies reviewed focusing on dementia or pre-dementia stages, 16 showed improvement in at least one 
cognitive or neuropsychiatric outcome measure. These data are promising and reinforce the potential clinical use of TMS and tDCS for the treatment of symptoms associated with Dementia and Parkinson's disease, despite the considerable variation in the parameters used for each technique.

Work on inducing behavioral changes at the cognitive level in healthy older adults in order to prevent or slow down age-associated cognitive changes is, however, scarce (Zimerman and Hummel 2010). Furthermore, extant studies show inconsistent results, and may be hardly comparable as they use different designs and outcome measurements (Elder and Taylor, 2014). Because of this, this systematic review is important, as it aims to clarify the contribution of TMS and tDCS as useful approaches to enhance cognitive function in healthy older adults.

\section{Neuromodulation techniques}

The use of non-invasive techniques to modulate brain function has a long tradition that dates back to the use of energy obtained from electric fishes (e.g., eels, torpedo fish, etc) to modify brain activity in order to alleviate headache or pain (Fregni and Pascual-Leone 2007). Over time, the use of brain stimulation by means of electrical currents became a therapeutic strategy to minimize the effects of mental disorders. In recent decades, NIBS has gained special relevance both in clinical and research settings, and techniques have been optimized with the ultimate goal of inducing long-lasting plastic changes at the sensory-motor and cognitive levels. The exponential interest in TMS and tDCS is mostly related to their safety and to the successful outcomes following the application of such techniques in clinical conditions (Miniussi et al. 2008; Dayan et al. 2013). Likewise, these techniques have also proven to be of major relevance in maintaining or improving the integrity of higher brain functions in the 
healthy aging process (Zimerman and Hummel 2010), when age-related neurobiological changes bring functional decline and impair quality of life.

TMS is based on the principles of electromagnetic induction, whereby a magnetic stimulator generates, stores and releases an electric current pulse through a coil, which in turn creates a magnetic field around its windings. The alternating magnetic field generated will penetrate the skull and the brain, inducing an electric field in the targeted cortical tissue. When applied over areas overlying the motor cortex, the TMS pulse elicits motor evoked potentials (MEP) in the contralateral limb. Because of this, TMS has been extensively used as a brain mapping method in an attempt to relate brain structure to cognitive and motor function (Romero et al. 2011). When applied repetitively (rTMS), it induces a modulation in the cortical excitability that depends on the frequency of stimulation: cortical excitability decreases in low-frequency rTMS (1 $\mathrm{Hz}$ or below), resulting in an inhibitory effect; and increases in high-frequency rTMS (5 $\mathrm{Hz}$ or above), producing an excitatory effect (Fregni and Pascual-Leone 2007). The effect of TMS on cortical activity depends, among other factors, on the shape of the stimulation coil, on the intensity of the magnetic field, on the position and angle of the coil over the cortex and on the direction of the induced cortical currents. Although TMS is considered a painless and safe technique when applied within safety guidelines, it presents some risks for adverse effects which need to be considered when designing a protocol (Rossi et al. 2009).

tDCS is a neuromodulation technique with polarity dependent effects. It consists of the application of a weak direct current in the scalp that flows between two or more electrodes - anode and cathode ( 2 electrodes in conventional tDCS vs. high definition (HD)-tDCS). In this process, low amplitude currents (up to $2 \mathrm{~mA}$ ) flow partially through the skull and penetrate the brain.Cortical modulation will depend on tDCS polarity: 
cathodal stimulation decreases cortical excitability by neuronal hyperpolarization, whereas anodal stimulation increases it by neuronal depolarization (Nitsche and Paulus 2011). Contrarily to other transcranial electric stimulation techniques (i.e., oscillatory tDCS, transcranial alternating current stimulation - tACS - and transcranial random noise stimulation - tRNS), tDCS uses a constant, and not fluctuant, current over a certain period of time (Filmer et al. 2014). The efficacy of tDCS-induced changes will primarily depend on the stimulation parameters, including polarity, size of the electrodes, the length of the stimulation period and the intensity of current. Several other factors may also influence the current distribution and, therefore, affect the efficacy of tDCS, including cranial and brain anatomy, such as skull thickness, densities of gray and white matter and cerebrospinal fluid volume and the presence of cerebral lesions. This is a safe technique, and only mild adverse effects have been reported (e.g., mild tingling or itching sensation, moderate fatigue or headache; Brunoni et al. 2011). Although the underlying mechanisms of action of NIBS techniques are not yet fully known, the effects of TMS and tDCS are probably related with plastic changes in the neural circuitry that are due to changes in long-term potentiation (LTP) and longterm depression (LTD; Hoffman and Cavus 2002). LTP/LTD effects are key mechanisms of many forms of experienced-dependent plasticity in the human brain, especially in what respects learning and memory. Both TMS and tDCS may induce long-term effects on cortical excitability, and the putative changes in LTP/LTD may have important implications for therapeutic interventions focusing on recovery of damaged or impaired brain functions (Ridding and Rothwell 2007).

\section{Methodology}


We conducted a review of the literature using exclusively the Pubmed research database to achieve a better standardization of results (Wong et al. 2006). Literature search and data extraction were conducted by A. M., and reviewed by J. A.. Literature search was performed using the mesh terms "transcranial", "magnetic stimulation", "direct current" and "aging" in the following conjunction "transcranial" AND "magnetic stimulation" OR "direct current" AND "aging". Studies were then screened individually according to our inclusion criteria, namely: (1) prospective experimental studies (clinical trials), where participants were subject to either (2) TMS or tDCS, focusing on (3) cognitive enhancement in (4) healthy older adults. Results were initially screened by title and after that by abstract review. Data extraction of relevant study information for studies meeting inclusion criteria was performed using a data extraction form to collect information on the sample characteristics, the intervention type, stimulation parameters, follow-up, cognitive domain and main outcomes.

After collecting and selecting the data, we conducted analysis to test and compare the results of the different studies, using the Comprehensive Meta-Analysis 2.0 software (Biostat Inc, Englewood, NJ, USA). We used Cohen's $d$ as an assessment of the effect size. We initially gathered the post-stimulation mean and standard deviation (SD) scores for each outcome in both groups (active and control/sham) from each paper. This was done in order to compare the mean changes between groups after the intervention. Given the heterogeneous sample and multiple cognitive outcomes, we focused the analysis to the primary outcome reported in the article, whenever possible. When it was not clearly stated, we used the first outcome reported by the authors. Additionally, when the means and SD where solely reported graphically, these data were estimated from the figures. Several studies contributed with more than one effect size, due to the different stimulation location (Cotelli et al. 2010, Ross et al. 2011, 
Manenti et al. 2013, Meinzer et al. 2014, Learmonth et al. 2015) or stimulation protocols (Fertonani et al. 2014, Sandrini et al. 2014). We excluded one study from the analysis (Berryhill and Jones, 2012), as there were no sufficient data available to compute the standardized mean differences. Subsequently, we computed the forest plot displaying the pooled effect size for the studies (Figure 2), using a random effects model. We chose a priori the random effects model as we expected considerable variability in the effect sizes, due to the diversity of protocols and features of the studies reviewed. Therefore, a common ("true") effect size (fixed random model) would not be appropriate. Furthermore, the random effects model is more conservative, as it lends relatively more weight to smaller studies and allows wider confidence intervals, thus reducing the likelihood of errors, namely type-II errors (Bolier et al. 2013).

We also used Comprehensive Meta-Analysis to examine any potential publication bias. The resulting funnel plot (Egger and Smith 1995) is shown in Figure 3. This method plots the standardized mean difference of each study against the respective standard error, allowing to visually check for publication bias. In the absence of bias, studies are expected to distribute equally to the left and right of the open diamond at the bottom (standard difference in means). To statistically assess any significant asymmetry, we applied the Egger's test (Egger et al. 1997) and the Begg and Mazumdar's correlation (Begg and Mazumdar 1994).

We also assessed the methodological quality of each study utilizing the Jadad Scale. This is a validated instrument widely used to measure the methodological quality of clinical trials, in which studies are scored based on the following three important methodological aspects broadly assumed to correlate with bias: randomization, blinding and dropouts/withdrawals (Clark et al. 1999). Total scores range from zero (poor quality) to five (the best quality). 


\section{Results}

With the initial search we identified a total of 209 references. From these, 28 were excluded as they were not identified as clinical trials. For the remaining 181, the titles were screened and only 22 met the selection criteria. Abstract review was then carried out, after which 11 studies were identified as meeting the inclusion criteria. All publications selected are in English. The main reasons for exclusion included failure to: (1) be a prospective experimental study, (2) be conducted in healthy older adults and (3) report cognitive outcomes. Experimental studies measuring motor or somatosensory outcomes, or neuroimaging studies measuring cortical activity with NIBS, with secondary behavioral cognitive outcomes were not included, as we were looking for studies assessing cognitive domains as primary outcomes. The procedural methodological summary for the trial selection process is outlined in the flow diagram of studies selection process in Figure 1.

Figure 1

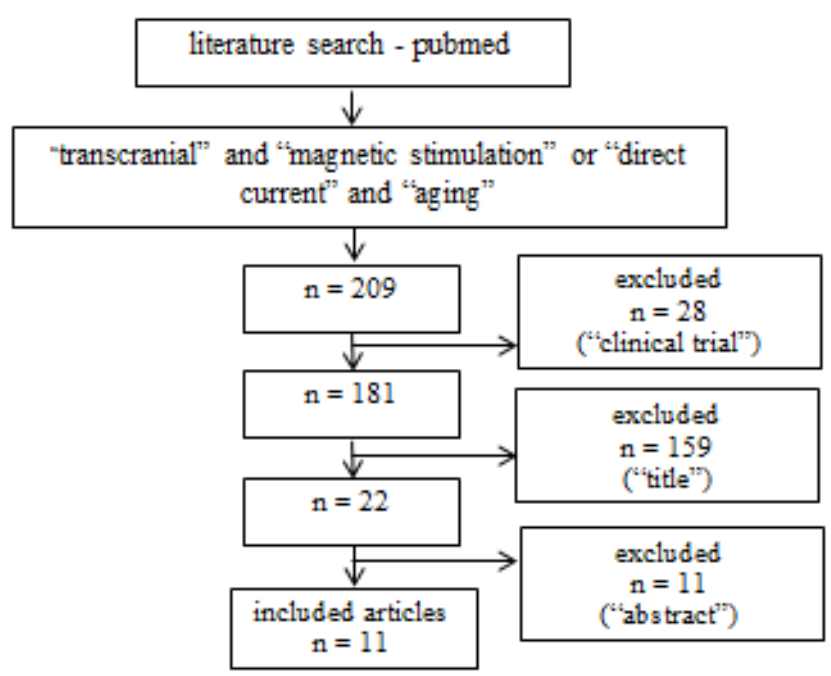

Figure 1 - Flow diagram of studies selection process 


\section{Synthesis of included studies}

The impact of NIBS techniques has been investigated in several cognitive domains. Among the eleven studies selected, five targeted memory as the main outcome (Ross et al. 2011, Berryhill and Jones 2012, Flöel et al. 2012, Manenti et al. 2013 and Sandrini et al. 2014), three targeted language (Cotelli et al. 2010, Meinzer et al. 2014 and Fertonani et al. 2014) and the following domains were address in one study each: inhibitory control (Kim et al. 2012), error awareness (Harty et al. 2014) and attention (Learmonth et al. 2015). To systematically describe the scope and components of each intervention study, specific features were extracted from each manuscript and are summarized in Table 1 . The design features and results of each study will be described narratively in brief, organized by cognitive domain.

\section{Impact on memory}

Memory has emerged as the most frequent cognitive domain investigated with NIBS. All of the five studies exploring memory performance used tDCS. More specifically, all the studies used anodal tDCS - i.e, the stimulation associated with an enhancement of cortical excitability. The intensity of tDCS was of 1mA in one study (Flöel et al. 2012) and 1.5mA in the remaining four studies. Stimulation time was variable across studies, with studies stimulating for 10min (Berryhill and Jones 2012), 15min (Ross et al. 2011; Sandrini et al. 2014), 20min (Flöel et al. 2012), or an adjustable amount of time according to the cognitive task being assessed (Manenti et al. 2013). Most studies targeted episodic memory (Flöel et al. 2012; Manenti et al. 2013; Sandrini et al. 2014), one of the main cognitive hallmarks of aging, but semantic memory (Ross et al. 2011) and working memory (Berryhil and Jones 2012) have also been studied. The predominant area of stimulation used in memory studies was the right 
or left dorsolateral prefrontal cortex (DLPFC; Berryhill and Jones 2012, Manenti et al. 2013; Sandrini et al. 2014), but the right temporoparietal cortex (TPC; Flöel et al. 2012), parietal cortex (PARC; Manenti et al. 2013) and the right or left anterior temporal lobe (ATL; Ross et al. 2011) have also been targeted in different studies. The cathode has been positioned as the reference electrode in the contralateral supraorbital region (Flöel et al. 2012, Manenti et al. 2013; Sandrini et al. 2014) or the contralateral cheek (Ross et al. 2011; Berryhill and Jones 2012). All the studies used the same type of sham procedure, which was a brief initial stimulation to produce a tingling sensation, which was decreased to 0 after 10s (Manenti et al. 2013; Sandrini et al. 2014), 15s (Ross et al. 2011), 20s (Berryhill and Jones 2012), or 30s (Flöel et al. 2012). Only one memory study included a younger group as control (Manenti et al. 2012). The majority of studies showed a positive effect for the stimulation condition - i.e., increased memory performance for the stimulation condition when compared to sham stimulation. In Berryhill and Jones (2012), tDCS was only beneficial in older adults with more years of education, showing a selective tDCS effect according to the education level. In Ross et al. (2011), the magnitude of the enhancing effect was similar in older and younger adults, but the lateralization of the effect was dependent on age. Similarly, Manenti et al. (2013) showed that tDCS facilitated episodic memory (i.e., increased memory retrieval), but only when tDCS was applied over the left regions (DLPFC or PARC), suggesting a lateralization of the processes (encoding/retrieval) associated with aging. Overall, the findings from the different studies are considered clinically relevant as they show the beneficial effects of tDCS on modulating memory performance, and suggest that this could be an interesting approach to study functional adaptation over the aging process, in both healthy older adults and in people with clinical memory conditions, as an attempt to reduce these cognitive deficits. 


\section{Impact on language}

The growing interest in evaluating new strategies to delay or counteract language deficits in healthy older adults led several authors to use either TMS or tDCS to explore effects on naming tasks. Excitatory rTMS was applied in one language study, with a frequency of $20 \mathrm{~Hz}$ and an intensity of $10 \%$ below the motor threshold (Cotelli et al. 2010). rTMS was delivered over the left and right DLPFC at the same time as the target picture was presented, in trains of $500 \mathrm{~ms}$ from the onset of the visual stimulus. tDCS was used in two other studies (Meinzer et al. 2014; Fertonani et al. 2014): in one anodal tDCS was used to test enhancement of cortical excitability (Fertonani et al. 2014), whereas in the other one cathodal and anodal tDCS were used to test both the enhancement and decrease of cortical excitability (Meinzer et al. 2014). tDCS intensity and stimulation duration differed by study: Meinzer et al. (2014) used an intensity of $1 \mathrm{~mA}$ for 30min, whereas Fertonani et al. (2014) used $2 \mathrm{~mA}$ for a variable time (according to the task). These studies targeted either the left DLPFC (Fertonani et al. 2014) or the right or left primary motor cortex (M1; Meinzer et al. 2014). The reference electrode (cathode) was positioned over contralateral locations: either the supraorbital region (Meinzer et al. 2014) or the shoulder (Fertonani et al. 2014). All the tDCS studies used the same type of sham procedure - brief initial stimulation to produce a tingling sensation followed by decreasing the administration to zero. However, they varied in the duration of initial stimulation, which was 10s in the Fertonani et al. (2014) study or 30s in Meinzer et al. (2014).

In the rTMS study, the sham condition was similar to the real stimulation condition, but stimulation was applied over the vertex and there was a piece of plywood under the coil. From the three studies testing language performance under NIBS in 
healthy older adults, only one used a control group of younger adults (Fertonani et al. 2014). The majority of the results suggest that real stimulation lead to an increase in accuracy in the naming task when compared to sham. Note that, in Cotelli et al. (2010), stimulation (rTMS) did not induce any effect on naming accuracy, but lead to faster naming latencies compared to sham. Interestingly, in the study by Fertonani et al. (2014), tDCS had a beneficial effect in the healthy older adult group only if applied during task execution (online tDCS), highlighting the importance of the activation of the language related neural network during stimulation. The overall positive findings from these studies assessing language performance in healthy older adults may have an important clinical impact, as they provide a rationale to explore NIBS techniques as novel intervention strategies to facilitate language processes in aphasia and other language impairments. Furthermore, these may be useful tools to complement traditional neuroimaging approaches in the investigation of age-dependent brain modifications.

\section{Impact on inhibitory control}

Based on the pre-existing evidence that rTMS facilitated inhibitory control in young adults, Kim et al. (2012) aimed to extend these findings to healthy older adults. They applied high-frequency $(10 \mathrm{~Hz})$ rTMS or sham stimulation to the left DLPFC in two experimental groups, for five consecutive days, and assessed changes in inhibitory control. Their results show that participants in the high-frequency rTMS group showed an enhancement in inhibitory control and a decreased in reaction times after rTMS when compared to the sham group (Kim et al. 2012). Interestingly, significant reaction times differences were obtained only for a subset of the experimental task (incongruent trials) in the active stimulation group. 


\section{Impact on error awareness}

Harty et al. (2014) set out to study whether the right DLPFC plays a causal role in supporting detection of performance errors ("error awareness"), and to evaluate the potential of tDCS for remediating awareness deficits in healthy older adults. The stimulating right DLPFC of healthy older adults with anodal tDCS, and tested whether this would lead to a significant improvement in error awareness as measured by an error awareness task (EAT). The influence of current polarity (anodal vs cathodal) and electrode location (left vs right hemisphere) was tested in a series of four trials. Intensity of tDCS stimulation was 1mA, and tDCS was applied over DLPFC while subjects performed a computerized test of error awareness. Stimulation duration depended on the length of the task. Overall, anodal tDCS over the right DLPFC was associated with a significant increase in the proportion of performance errors that were consciously detected, compared with sham and the cathodal condition (Harty et al. 2014). Furthermore, cathodal stimulation did not induce any behavioral changes relative to sham. No change in performance was observed when the left hemisphere was targeted.

\section{Impact on attention}

The effect of NIBS in spatial attention in healthy older adults has recently been investigated by Learmonth et al. (2015). Specifically, the experiment consisted of the application of either $1 \mathrm{~mA}$ anodal tDCS for 15 minutes to the right and left posterior parietal cortex or a sham protocol in young and healthy older adults during a titrated lateralized visual detection task. The idea was to use anodal tDCS to facilitate visual detection in the contralateral visual field. Results showed no differences in the effects of stimulation between young and older adults. Instead, the effects of anodal tDCS were state-dependent (i.e. related to task performance at baseline / influenced by an 
individual's baseline task performance), whereas age did not differentially affect response (Learmonth et al. 2015).

\section{Adverse effects of NIBS}

The main adverse effects of stimulation reported in the studies selected for this review are also summarized in Table 1, along with the parameters of stimulation and main results. No major adverse effects were reported following the application of TMS or tDCS, and no participants dropped out because of adverse events. The two studies that used TMS did not include a report on the potential adverse effects after stimulation, although one of these papers (Cotelli et al. 2010) emphasized that the stimulation protocol was in line with the safety recommendations proposed by Rossi et al. (2009). Most of the studies using tDCS referred to the safety profile of the technique based on existing guidelines or safety procedures, choosing stimulation parameters within the safety limits. Itching was repeatedly described as the most frequent adverse sensation, and was reported in five studies (Manenti et al. 2013, Sandrini et al. 2014, Fertonani et al. 2014, Harty et al. 2014; Learmonth et al. 2015). Burning sensation, discomfort, pitching, tingling, irritation and pain are also in the list of most frequent adverse effects across studies. Two of the studies (Ross et al. 2011; Berryhill and Jones 2012) do not mention any assessment of adverse effects, and in another study the authors did not find any noteworthy adverse effect to be reported (Meinzer et al. 2014). Overall, the experienced perceptual sensations started at the beginning of the experiment, did not last long and did not affect task performance in the tDCS or sham conditions. Flöel et al. (2012) used other measures to assess potential adverse effects derived from stimulation, namely heart rate, blood pressure and mood rating. These measures did not seem to show any significant adverse effect. 


\section{Results of the quantitative analysis}

Our assessment of the magnitude of the effects of the different studies was based on 10 articles with 17 effect sizes. Considering that an effect size of 0.2 is small, 0.5 is medium/moderate and an effect over 0.8 is considered to be large (Sullivan and Feinn, 2012), we found an overall mean moderate to large positive effect on cognitive outcomes post-intervention (tDCS or rTMS; mean effect size of $0.57,95 \%$ CI, 0.34 , $0.79, p<0.05$; see Figure 2). We also found moderate heterogeneity across studies $\left(\mathrm{I}^{2}=\right.$ 47.5).

To assess publication bias on our sample, we generated a funnel plot displaying the effect sizes from individual studies (horizontal axis) against the standard error (vertical axis) (Figure 3 about here). As can be seen in Figure 3, the distribution of studies is relatively asymmetric, with more studies laying to one of the sides of the plot, which suggests the likelihood of publication bias. Additionally, one of the studies lies outside the funnel. The Egger's regression intercept was 3.65 (two-tailed $p=0.03$ ) and the Begg and Mazumdar's correlation was 0.44 (two-tailed $p=0.01$ ). In sum, the funnel plot and the significant correlation tests suggest that there is publication bias. However, the small number of studies makes it difficult to reach any strong conclusion.

\section{Quality assessment}

To assess the methodological quality of the studies, we adapted the criteria suggested by Jadad et al. (1999) which focus on randomization, blinding and dropouts/withdrawals. A summary of the Jadad individual assessments for each trial is shown in Table 2. Seven of the studies were reported as randomized trial studies (Ross et al. 2011, Flöel et al. 2012, Manenti et al. 2013, Sandrini et al. 2014, Meinzer et al. 2014, Kim et al. 2012, Harty et al. 2014), although the randomization and allocation 
processes are not fully described. The majority of studies were reported as blinded (Flöel et al. 2012, Manenti et al. 2013, Sandrini et al. 2014, Fertonani et al. 2014, Kim et al. 2012; Harty et al. 2014). However, all but two of these studies did not appropriately described how this was implemented (Flöel et al. 2012, Sandrini et al. 2014). Finally, only four studies reported whether there were dropouts or withdrawals, along with the reasons for exclusion (Ross et al. 2011, Flöel et al. 2012, Meinzer et al. 2014; Harty et al. 2014). The other studies failed to mention whether and how there were any dropouts or withdrawals.

\section{Discussion}

In the past 20 years, noninvasive brain stimulation has been extensively used in humans to modulated different motor and cognitive functions (Reis et al. 2008). Techniques such as TMS and tDCS have improved our understanding of brain-behavior interactions and allowed us to explore possible casual links between changes in activity in specific brain areas and behavior (Siebner and Rothwell 2003). In this review we explored whether the use of NIBS techniques, specifically TMS and tDCS, leads to positive outcomes when trying to improve cognitive functioning in healthy older adults. We conducted a thorough literature review and collected evidence from clinical trials using either TMS or tDCS as a strategy to impact cognitive function in healthy older adults. Despite considerable variability in the targeted cognitive domains, design features and outcomes of the selected studies, the results from our systematic review generally show that performance in cognitive tasks is enhanced in healthy older adults, and that these individuals uniformly benefit from the use of NIBS techniques across studies. Specifically, the meta-analysis of the studies included in this review showed a significant pooled effect size of 0.57 , which is likely to be clinically significant. 
These data are consistent with previous studies conducted in other populations, namely young adults, that also show positive effects of the use of NIBS in cognitive performance (Clark et al. 2011, Simis et al. 2013; Zibly et al. 2014). But are these techniques equally effective when applied to young and healthy older adults? Some studies seem to suggest that this is not the case - the effects of TMS and tDCS may be more pronounced in young adults than in healthy older adults (Manenti et al. 2013, Fertonani et al. 2014). Nevertheless, other studies suggest that neuromodulation promotes similar effects in young and healthy older adults (Ross et al. 2011, Learmonth et al. 2015). One possible explanation for the putative diminished effect of NIBS in healthy older adults, when compared to the effects on young adults, relates to individual network changes that are inherent to the aging process, and that determine what the optimal areas for stimulation are when targeting different cognitive and motor functions (Antonenko and Flöel 2013). Recent studies have demonstrated that healthy older adults show reduced functional connectivity within the typical default brain networks, and poorer behavioral performance in various cognitive domains that is associated with white matter defects (Andrews-Hanna et al. 2007; Geerligs et al. 2014). It is possible, then, that the weaker effects observed in healthy older adults in some studies are due to functional reorganization in the aging brain (Harty et al. 2014). In future studies, the use of NIBS techniques should be preceded by a mapping of the individual neural networks that support the targeted domain in order to maximize the success of the intervention.

An important issue to bear in mind when design the kinds of interventions reviewed here is related with the fact that the effects of NIBS may differ substantially by educational level of the participants (Reis et al. 2008). For instance, Berryhill and Jones (2012) showed that performance on memory tasks after NIBS differed according to the education level of the participant - tDCS was only beneficial in older adults with 
more education. The psychological and physiological benefits of education on cognition in healthy older adults, and specifically in the memory domain, have long been established.Studies suggest that an high-level of formal education exerts a protective effect against cognition decline (Kubzansky et al. 1998). Interestingly, prior studies on the use of tDCS show that effects may not be observed when baseline levels of performance are already high (Kang et al. 2009), and that the facilitatory effects of the tDCS may be more pronounced when there are greater levels of impairment (Hummel et al. 2006), suggesting a potential ceiling effect in highly educated samples (Wang et al. 2009).

Another set of aspects that will influence the outcome of an intervention is related with the structure, design and quality of the clinical trial to be implemented. One such aspect is the number of sessions and follow-ups that are included in a trial, and whether and how these can lead to long-lasting effects on performance. So far, studies mostly focused on the ability of neuromodulation techniques to elicit relatively shortlasting improvements in humans. A recent review on the effects of tDCS has demonstrated that single-session studies have no reliable effect on cognition in healthy adults (Horvath et al. 2015). Additionally, it has been shown that five consecutive sessions of anodal tDCS can lead to gains that can persist for several weeks after the stimulation period (Boggio et al. 2012). However, Sandrini et al. (2014) suggested that these long-lasting (e.g., one month) behavioral effects after tDCS stimulation might actually not be due to the effect of tDCS, but to the effect of practicing existing memories. Regarding rTMS effects, there is currently a limited understanding of how long the effects of HF-rTMS on cognitive function may persist (Guse et al. 2010, Rutherford, Lithgow and Moussavi, 2015). It has been suggested that longer stimulation trains would generally induce more lasting effects (Eisenegger et al. 2008). 
The methodological quality of the clinical trial is also an important aspect to consider. Overall, and according to the Jadad scale, the studies included in this review revealed low methodological quality. For instance, if a trial lacks proper randomization, the treatment groups will likely not be balanced, decreasing the validity of the study (Bridgman et al. 2003). On the other hand, the randomization process may introduce biases if the mechanisms for generating the randomization sequences or the means for ensuring randomization concealment are not fully appropriate. In our results, only seven studies were reported as randomized trials. In all of them, the description of the randomization procedures was not fully or adequately presented.

As for blinding, if studies are not appropriately blinded, the results may be inflated due to conscious or unconscious biases, either from the investigator or the research subjects (Bridgman et al. 2003). Blinding is mentioned in most of the studies we reviewed, but most of them did not appropriately report how blinding was implemented. In fact, only two studies (Flöel et al. 2012; Sandrini et al. 2014) presented a complete description of their blinding procedures. Blinding in interventions that use neuromodulation may be hard to achieve. For instance, not all the research teams have access to rTMS sham coils. Nevertheless, alternative methods should be implemented, such has holding the stimulation coil in a different angle. The study by Cotelli et al. (2010) is a great example of an alternative strategy to implement blinding - in this study, a piece of plywood was applied to the coil under sham stimulation to prevent the magnetic fields from penetrating the cortex.

The description of dropouts and withdrawals is another important methodological feature that may correlate with bias (Bridgman et al. 2003). The fate of participants in the trial must be described and reasons for dropout fully disclosed, so that the analysis of missing data can be adequately performed and risk of biases 
reduced. Only four of the studies in our review presented a description of dropouts and withdrawals. The remaining studies did not provide any direct reference to the final sample included in the analysis.

As such, the assessment of the quality of the studies included in this review, and potentially of most of the studies that include neuromodulation, cannot be fully accomplished, which may be one of the potential pitfalls and limitations associated with clinical trials that use neuromodulation techniques. It is also important to note that these trials are exploratory in nature, and therefore subject to other biases. For instance, as these studies typically include small sample sizes, selection and sampling bias may occur and, therefore, generalizability may be limited. Furthermore, analyzing the relationship between quality assessment and the direction of the outcomes - i.e., positive or negative outcomes - would be interesting as a way of understanding how the methodological aspects are related with the overall study results. According to Chiavetta et al. (2014), potential differences in methodological quality between trials with positive and negative outcomes may lead to positive trials with biased results, or to the discrimination of negative trials. Moreover, by acknowledging the importance of such methodological features, investigators may become aware of how imperative it is to assess the quality of published trials, and re-think the design features to implement in their own research work.

A final set of aspects that is important to mention deals with technical and methodological issues that are central to NIBS techniques. The results presented in this review suggest that tDCS and TMS are indeed promising strategies in the context of cognitive enhancement in healthy older adults. There are, however, some aspects of the selected studies that may have more or less impact in the success of an intervention using a NIBS technique. Overall, rTMS was used in two studies (18\%), whereas tDCS 
was used in nine studies (82\%). The locus of stimulation and the targeted cognitive domains were also different across studies. The DLPFC was the cortical area that was most explored with NIBS for the cognitive domains of memory, language, error awareness and inhibitory control. Moreover, parietal cortex was also targeted to impact on memory and attention, the ATL and the right TPC to impact on memory, and M1 was targeted to assess the language domain. Another aspect that differed across studies was intensity and frequency of stimulation. Both rTMS protocols applied excitatory stimulation, with frequencies ranging from $10 \mathrm{~Hz}$ to $20 \mathrm{~Hz}$, but their sham stimulation set up was different: in one study the angle of stimulation was changed and only one edge of the coil rested on the scalp (Kim et al. 2012), whereas on the other study, the location of stimulation was not the same as the active stimulus, and a piece of plywood was applied to the coil (Cotelli et al. 2010). tDCS stimulation parameters also varied across studies in several items. The polarity of stimulation was fairly consistent across studies - the target area was excited (anodal electrode), whereas the reference area was inhibited (cathode electrode). In two studies, both anode and cathode worked as active stimulation conditions so that one could compare the effect of these conditions within groups (Meinzer et al. 2014; Harty et al. 2014). Interestingly, there is larger variability in the intensity of stimulation that was used. The intensities used ranged from $1 \mathrm{~mA}$ to $2 \mathrm{~mA}$. Stimulation duration also varied considerably across studies, with authors using fixed periods up of 10, 15, 20 or $30 \mathrm{~min}$. All studies used the same type of sham procedure (i.e., a brief initial and sometimes final stimulation that produced a tingling sensation). The ramp up and down period also varied across studies, with periods of onset and offset of stimulus of 10, 15, 20 and 30 seconds. All protocols used saline soaked sponge electrodes that were variable in size. 
The findings from the different studies that were reviewed here showed us that despite the different protocols and stimulation parameters, both tDCS and TMS can modulate performance in several cognitive domains. Nevertheless, it is important to note that stimulating one cortical area may result in direct neural changes (and associated behavioral changes) on that particular area, and/or affect interconnected areas that then lead to observed behavioral changes (Siebner et al. 2009). One important consideration to highlight is the possible impact of brain anatomy and connectivity in the mechanism through which TMS and tDCS work. As the surface of the brain is not a smooth sphere, hyperpolarization of a set of neurons in a specific structure (i.e., gyrus) may result in depolarization in other structures. This is especially relevant in healthy older adults due to the age-related cortical thinning and other anatomical changes. Although this is an important issue that deserves discussion, it is often neglected. In fact, in our sampled studies, few mention this possibility when describing and discussing their results. For instance, Sandrini et al. (2014) mentioned the possibility that their tDCS-induced effects may not be exclusively due to activation of the targeted local region, but also to the activation of remote interconnected regions. This has also been suggested in a similar study, in young participants, where rTMS was used over the right DLPFC (Sandrini et al. 2013). The fact that rTMS facilitates a network rather than a single area is also discussed by Cotelli et al. (2010). This is consistent with a large number of focal lesion and neuroimaging studies which demonstrate that cognitive abilities depend on a wide and complex network of associated regions, and are not just associated to a single area (Damasio et al. 2001; DeLeon et al. 2007, Almeida, Fintzi and Mahon 2013, Mahon, Kumar and Almeida 2013, Garcea and Mahon 2014).

An interesting question that may arise from this review of the literature is whether some NIBS techniques may be more suitable to study cognitive changes in 
healthy older adults. Whereas TMS induces hyperpolarization or depolarization of the neurons and the firing of an action potential, tDCS modulates existing spontaneous activity. Therefore, one can argue that tDCS may be a more interesting technique to study cognitive enhancement in healthy older adults, as it causes spontaneous neuronal firing in neurons that are already active. On the other hand, tDCS may not be the most appropriate approach for individuals with brain or skull lesions, because these lesions are expected to distort the flow of current, raising safety and efficacy concerns. Because TMS uses motor-evoked potentials to index the intensity of stimulation, it can be adjusted in a patient specific manner, and take in account lesions and other situations that can disrupt the flow of the current in tDCS (Priori et al. 2009). One of the strengths of tDCS, over TMS, as a research method, is that it can be easily and adequately shammed. Additionally, it has the advantage of being easier to apply concomitantly with behavioral tasks, such as cognitive training. Another feature that figures critically in the success of NIBS stimulation is related with particular physical characteristics of the equipment used. Specifically, it is widely accepted that different shapes of the TMS coil, and the sizes of the tDCS electrodes can lead to variations on how focal the stimulation is (Rossi et al., 2009; DaSilva et al. 2011). In our results, there is some consensus on the type of device used for rTMS - all the studies use a 70mm figure eight coil. This coil shape allows for a relatively more focal stimulation of the target cortical regions (Rossi et al. 2009). On the other hand, there was no consensus on the size of the tDCS electrodes to be used. According to DaSilva et al. (2011), decreasing the diameter of the electrodes may result in more focal stimulation. Meinzer et al. (2014) also emphasize the potential impact of the size of the active and reference electrodes on the behavioral outcome. The larger the electrodes, the larger the likelihood of stimulating regions in the vicinity of the region of interest. Thus, by producing excitability changes 
in different regions, we are recruiting additional cortical networks which may have an impact in the outcome as well. Furthermore, it is important to note that these techniques also modulate functional connectivity between remote, but connected brain regions. Therefore, it is difficult to tie the effects of tDCS to a specific brain region.

A rather important topic that necessarily has to be taking in consideration when devising an intervention that uses NIBS techniques is the implementation of safety procedures. TMS and tDCS are noninvasive in nature, but both stimulation techniques are associated with potential risks that demand certain safety precautions (Sparing and Mottaghy 2008). Because both techniques rely on changes of cortical excitability, the major concern is the possibility that NIBS stimulation can trigger a seizure. It is important to note, however, that a seizure induced by the use of tDCS has never been reported in all the years that this technique has been used. In the case of TMS, there were some instances where a participant experienced a seizure. Importantly, these instances happened when the safety guidelines were not being followed (Rossi et al. 2009). If the research team follows the appropriate guidelines and recommendations that have been proposed for both techniques (TMS: Rossi et al. 2009; tDCS: Woods et al. 2015), then the techniques can be applied safely with minimal or no adverse effects. In our review, no major adverse effects were reported. In the studies that describe adverse effects, these are minor and in agreement with the available literature (Poreisz et al. 2007; Rossi et al. 2009). One potential problematic factor is that the lack of reporting in the manuscripts does not necessarily imply an absence of adverse effects. For instance, minor adverse effects may have not been extensively reported by the subject, or the authors may have not included them in the final manuscript.

One final topic that does not figure in our review but is a very promising avenue in the use of NIBS to enhance and ameliorate cognitive functioning and decline in 
healthy older adults in the combination of NIBS techniques and cognitive training programs. In the past couple of years, cognitive training programs have been identified as the most favorable method to enhance brain plasticity, with several brain imaging studies revealing training-induced plasticity in the healthy human brain (Erickson et al. 2007, McNab et al. 2009). Recently, the cumulative facilitatory effects of cognitive training used together with NIBS started to be explored, in both clinical populations and healthy older adults. The rationale for this combination lies on the possibility of enhancing plasticity and strengthening memory networks with both techniques. To understand the direction and extent of the use of NIBS with cognitive training in healthy older adults, we conducted a brief review on Pubmed using the terms "tms" OR "tdcs" AND "cognitive training" AND "older adults". We found a total of 4 references, including 2 experimental studies, a review of the literature and a protocol for a randomized controlled trial.

The experimental studies (Park et al., 2014; Jones et al., 2015) explored the impact of tDCS combined with cognitive training on working memory performance in cognitively intact older adults. In Park et al. (2014), 40 healthy older adults were randomly assigned to either active (anodal) or sham tDCS bilaterally over the prefrontal cortex. Participants went through 10 sessions where they first underwent tDCS (sham or active) and then performed computerized working memory cognitive training tasks. The authors collected data on the accuracy of a verbal working memory task and on the digit span forward test. By the end of the intervention (i.e., 10 sessions), performance in both the verbal working memory task and the digit span forward test improved significantly in the active tDCS group as compared to sham group. Importantly, these effects remained stable at follow-up (4 weeks later) for the verbal working memory task. In Jones et al. (2015), 72 participants underwent 10 sessions of active or sham tDCS in the 
right prefrontal, parietal, or prefrontal/parietal (alternating) cortices. Anodal tDCS was delivered for 10 minutes, at an intensity of $1.5 \mathrm{~mA}$. Following tDCS, participants completed a computerized cognitive training protocol that focused on visual and verbal working memory. In some of the sessions (first, last and at 1 month follow-up), participants also performed transfer working memory. Overall, the four groups benefited from the intervention (tDCS + cognitive training), but significant improvement at follow-up was only observed in the active tDCS group.

The results from these studies suggest that the combination of NIBS and other cognitive enhancement strategies (e.g., computerized cognitive training) can counteract cognitive decline in healthy older adults. Similarly to what we found in our main systematic review, the technique used in the majority of studies was tDCS. Perhaps the preference for tDCS over TMS is related with its mechanism of action involving the modulation of spontaneous neuronal activity, its safety profile, and ease of application. While these preliminary findings are encouraging, some important questions need to be addressed. Firstly, long-term positive effects have only been tested at one month after the intervention in both studies. Thus, we should also consider the possibility that these results are not due to the effect of stimulation, but rather a consequence of the repeated practice of memory training in the intervention period (learning and performance effects from practice). It would be important to measure whether the effects remain after a longer period without stimulation (i.e., testing 6 months after stimulation). Another important aspect to consider is the need of including a control group receiving tDCS in the absence of cognitive training protocols. This would clarify whether the benefits of combining both techniques are greater than tDCS provided alone. None of the studies explored this question (Park et al., 2014; Jones et al., 2015). Furthermore, it is unclear whether the reported effects can transfer to other cognitive domains (e.g., attention, 
processing speed).

\section{Limitations and future studies}

One of the major outcomes of our review is that there is still a long way to go before we reach optimal and consensual protocols for the use of NIBS in the effort of counteracting age-related cognitive decline. The major sources of bias that we observed in the studies we reviewed are related with the appropriateness of the randomization process and the blinding method, the extreme variability among protocols, sample features (i.e., dropouts and withdrawals) and sample subjectivity (i.e., level of education and cognitive abilities). Field-wide efforts should be made in order to establish goldstandards and guidelines to be followed by future research. Similarly, it would be extremely helpful to have outcomes assessed by the same evaluation process so that the different interventions can easily be compared to one another. Reaching a consensus on the optimal design and parameters for the different cognitive functions would be an important step towards an effective evaluation of therapeutic approaches that use neuromodulation, regardless of the type and/or process involved.

Additionally, the criteria used for participant inclusion in the healthy older adult experimental group varied widely across papers. For instance, Ross et al. (2011) used very strict inclusion criteria for their healthy older adult group. These criteria included scoring above a predefined cut-off in a set of selected neuropsychological assessment tools. Cotelli et al. (2010), employed a more lenient set of criteria to screen potential participants. These included a standard health history questionnaire and a brief neurological assessment. These differences in inclusion and exclusion criteria may lead to important differences in the target sample used in each study and may hamper generalization of the results. 
Also, stimulating over-recruited areas should be further explored as well, to explore the role of the targeted areas over specific cognitive domains and its subsequence age-related change. The combination of brain stimulation with other brain mapping techniques holds great potential to provide even more valuable advances in our understanding of human cognition. The functional changes in cortical reactivity and effective connectivity induced by cognitive plasticity should be evaluated using NIBS with other techniques (i.e., EEG, fMRI) before, during and after the interventions.

Finally, there are some additional limitations from this review that are worth mentioning. Some studies did not report the sizes of their effects nor the necessary data to calculate them directly. In those situations the data used for the meta-analysis was estimated from the figures provided in those papers. This clear lends some variability to the data that entered the calculations of the overall measures of effect size.

\section{Conclusion}

Overall, the use of TMS and tDCS in interventions that focus on counteracting cognitive decline in healthy older adults has met with promising results. These results can be seen as preliminary steps towards the development of tools to enhance cognitive performance in healthy older adults, as well as other clinical populations affected by cognitive decline. Further work is required in order to achieve a better standardization of the optimal parameters to achieve positive outcomes, as well as to determine whether these techniques can promote long-term cognitive improvement. Another important avenue of research is to search for new strategies, or combination of strategies, that can enhance or maintain cognitive functioning in healthy older adults. 


\section{References}

Almeida, J., Fintzi, A. R., \& Mahon, B. Z. (2013). Tool manipulation knowledge is retrieved by way of the ventral visual object processing pathway. Cortex, 49(9), 2334-2344.

American Psychiatric Association (2013). Diagnostic and Statistical Manual of Mental Disorders, Arlington. doi:10.1176/appi.books.9780890425596.744053

Andrews-Hanna, J.R., Snyder, A.Z., Vincent, J.L., Lustig, C., Head, D., Raichle, M.E., \& Buckner, R. L. (2007). Disruption of large-scale brain systems in advanced aging. Neuron, 56(5), 924-935.

Angevaren, M., Aufdemkampe, G., Verhaar, H.J.J., Aleman, A. \& Vanhees, L. (2008). Physical activity and enhanced fitness to improve cognitive function in older people without known cognitive impairment. Cochrane database Syst. Rev. doi:10.1002/14651858.CD005381.pub3

Antonenko, D. \& Flöel, A. (2013). Healthy aging by staying selectively connected: A mini-review. Gerontology, 60(1), 3-9.

Balconi, M. \& Ferrari, C. (2012). rTMS stimulation on left DLPFC increases the correct recognition of memories for emotional target and distractor words. Cognitive. Affective and Behavioral Neuroscience, 12(3), 589-598.

Bamidis, P.D., Vivas, a. B., Styliadis, C., Frantzidis, C., Klados, M., Schlee, W., ... \& Papageorgiou, S. G. (2014). A review of physical and cognitive interventions in aging. Neuroscience and Biobehavioral Reviews, 44, 206-220. 
Berryhill, M.E. \& Jones, K.T. (2012). tDCS selectively improves working memory in older adults with more education. Neuroscience Letters, 521(2), 148-151.

Bishop, N. A, Lu, T. \& Yankner, B. (2010). Neural mechanisms of ageing and cognitive decline. Nature, 464, 529-535.

Boggio, P. S., Ferrucci, R., Mameli, F., Martins, D., Martins, O., Vergari, M., ... \& Priori, A. (2012). Prolonged visual memory enhancement after direct current stimulation in Alzheimer's disease. Brain Stimuation, 5(3), 223-230.

Bolier, L., Haverman, M., Westerhof, G. J., Riper, H., Smit, F., \& Bohlmeijer, E. (2013). Positive psychology interventions: a meta-analysis of randomized controlled studies. BMC Public Health, 13(1), 1.

Borenstein M, Hedges L, Higgins J, Rothstein H. Comprehensive Meta-analysis Version 2, Biostat, Englewood NJ [2005]).

Braver, T.S. \& Barch, D.M. (2002). A theory of cognitive control, aging cognition, and neuromodulation. Neuroscience and Biobehavioral Reviews, 26(7), 809-817.

Bridgman, S., Dainty, K., Kirkley, A. \& Maffulli, N. (2003). Practical aspects of randomization and blinding in randomized clinical trials. Arthroscopy, 19(9), 1000-1006.

Brunoni, A.R., Amadera, J., Berbel, B., Volz, M.S., Rizzerio, B.G. \& Fregni, F. (2011). A systematic review on reporting and assessment of adverse effects associated with transcranial direct current stimulation. Internacional Journal of Neuropsychopharmacoly, 14(8), 1133-1145. 
Brunoni, A. R., Nitsche, M. A., Bolognini, N., Bikson, M., Wagner, T., Merabet, L., ... \& Ferrucci, R. (2012). Clinical research with transcranial direct current stimulation (tDCS): challenges and future directions. Brain Stimulation, 5(3), 175-195.

Chiavetta, N.M., Martins, A.R.S., Henriques, I.C.R. \& Fregni, F. (2014). Differences in methodological quality between positive and negative published clinical trials. Journal of Advanced Nursing, doi:10.1111/jan.12380.

Clark, H. D., Wells, G. A., Huët, C., McAlister, F. A., Salmi, L. R., Fergusson, D., \& Laupacis, A. (1999). Assessing the quality of randomized trials: Reliability of the Jadad scale. Controlled Clinical Trials, 20(5), 448-452.

Clark, V.P., Coffman, B.A., Trumbo, M.C. \& Gasparovic, C. (2011). Transcranial direct current stimulation (tDCS) produces localized and specific alterations in neurochemistry: a ${ }^{1} \mathrm{H}$ magnetic resonance spectroscopy study. Neuroscience Letters, 500(1), 67-71.

Cotelli, M., Manenti, R., Rosini, S., Calabria, M., Brambilla, M., Bisiacchi, P. S., ... \& Miniussi, C. (2010). Action and object naming in physiological aging: An rTMS study. Frontiers in Aging Neuroscience, 2(151), 1-7.

DaSilva, A.F., Volz, M.S., Bikson, M. \& Fregni, F. (2011). Electrode positioning and montage in transcranial direct current stimulation. Journal of Visualized Experiments, 51, 1-9.

Damasio, H., Grabowski, T.J., Tranel, D., Ponto, L.L., Hichwa, R.D. \& Damasio, R. (2001). Neural correlates of naming actions and of naming spatial relations. Neuroimage 13(6), 1053-1064. 
Dayan, E., Censor, N., Buch, E.R., Sandrini, M. \& Cohen, L.G. (2013). Noninvasive brain stimulation: from physiology to network dynamics and back. Nature Neurosciences, 16(7), 838-44.

DeLeon, J., Gottesman, R. F., Kleinman, J. T., Newhart, M., Davis, C., Heidler-Gary, J., ... \& Hillis, A. E. (2007). Neural regions essential for distinct cognitive processes underlying picture naming. Brain, 130(5), 1408-1422.

Demirtas-Tatlidede, A., Vahabzadeh-Hagh, A.M. \& Pascual-Leone, A. (2013). Can noninvasive brain stimulation enhance cognition in neuropsychiatric disorders? Neuropharmacology, 64, 566-578.

Den Heijer, T., van der Lijn, F., Koudstaal, P.J., Hofman, A., van der Lugt, A., Krestin, G.P. (2010). A 10-year follow-up of hippocampal volume on magnetic resonance imaging in early dementia and cognitive decline. Brain, 133, 1163-72.

Begg, C. B., \& Mazumdar, M. (1994). Operating characteristics of a rank correlation test for publication bias. Biometrics, 1088-1101.

Egger, M., \& Smith, G. D. (1995). Misleading meta-analysis. BMJ: British Medical Journal, 311(7007), 753.

Egger, M., Smith, G. D., Schneider, M., \& Minder, C. (1997). Bias in meta-analysis detected by a simple, graphical test. Bmj, 315(7109), 629-634.Eisenegger, C., Treyer, V., Fehr, E. \& Knoch, D. (2008). Time-course of "off-line" prefrontal rTMS effects - a PET study. Neuroimage, 42(1), 379-384.

Elder, G. \& Taylor, J-P. (2014). Transcranial magnetic stimulation and transcranial direct current stimulation: treatments for cognitive and neuropsychiatric 
symptoms in the neurodegenerative dementias? Alzheimer's Research \& Therapy, 6(9), 74.

Erickson, K. I., Colcombe, S. J., Wadhwa, R., Bherer, L., Peterson, M. S., Scalf, P. E., ... \& Kramer, A. F. (2007). Training-induced plasticity in older adults: effects of training on hemispheric asymmetry. Neurobiology of aging,28(2), 272-283.

Fertonani, A., Brambilla, M., Cotelli, M. \& Miniussi, C. (2014). The timing of cognitive plasticity in physiological aging: A tDCS study of naming. Frontiers in Aging Neuroscience. 6(131), 1-9.

Filmer, H.L., Dux, P.E. \& Mattingley, J.B. (2014). Applications of transcranial direct current stimulation for understanding brain function. Trends in Neurosciences, 37(12), 1-12.

Flöel, A., Suttorp, W., Kohl, O., Kürten, J., Lohmann, H., Breitenstein, C., \& Knecht, S. (2012). Non-invasive brain stimulation improves object-location learning in the elderly. Neurobiology of Aging, 33(8), 1682-1689.

Fregni, F. \& Pascual-Leone, A. (2007). Technology insight: noninvasive brain stimulation in neurology-perspectives on the therapeutic potential of rTMS and tDCS. Nature Clinical Practice Neurology, 3(7), 383-393.

Friedman, D. (2013). The cognitive aging of episodic memory: a view based on the event-related brain potential. Frontiers in Behavioral Neuroscience. 7, 111.

Garcea, F.E., \& Mahon, B.Z. (2014). Parcellation of left parietal tool representations by functional connectivity. Neuropsychologia, 60, 131-143. 
Geerligs, L., Maurits, N.M., Renken, R.J. \& Lorist, M.M. (2014). Reduced specificity of functional connectivity in the aging brain during task performance. Human Brain Mapping, 35(1), 319-30.

Glisky, E.L. (2007). Changes in cognitive function in human aging. In D- R. Riddle (Ed.), Brain Aging: Models, Methods, and Mechanisms (pp. 3-20). WinstonSalem, NC: CRC Press.

Grady, C. (2012). Trends in neurocognitive aging. Nature Reviews Neuroscience. 13(7), $491-505$.

Guse, B., Falkai, P. \& Wobrock, T. (2010). Cognitive effects of high-frequency repetitive transcranial magnetic stimulation: a systematic review. Journal of Neural Transmission. 117(1), 105-122.

Hartwigsen, G. (2014). The neurophysiology of language: Insights from non-invasive brain stimulation in the healthy human brain. Brain Language, 148, 81-94.

Harty, S., Robertson, I. H., Miniussi, C., Sheehy, O. C., Devine, C. A., McCreery, S., \& O'Connell, R. G. (2014). Transcranial direct current stimulation over right dorsolateral prefrontal cortex enhances error awareness in older age. Journal of Neuroscience, 34(10), 3646-52.

Hoffman, R.E. \& Cavus, I. (2002). Slow transcranial magnetic stimulation, long-term depotentiation, and brain hyperexcitability disorders. American Journal of Psychiatry, 159(7), 1093-1102. 
Horvath, J.C., Forte, J.D. \& Carter, O. (2015). Quantitative Review Finds No Evidence of Cognitive Effects in Healthy Populations from Single-Session Transcranial Direct Current Stimulation (tDCS). Brain Stimulation, 8(3), 535-550.

Hummel, F.C. \& Cohen, L.G. (2006a). Non-invasive brain stimulation: a new strategy to improve neurorehabilitation after stroke? Lancet Neurology, 5(8), 708-712.

Hummel, F. C., Voller, B., Celnik, P., Floel, A., Giraux, P., Gerloff, C., \& Cohen, L. G. (2006b). Effects of brain polarization on reaction times and pinch force in chronic stroke. BMC Neuroscience, 7, 73.

Imhof, A., Kövari, E., von Gunten, A., Gold, G., Rivara, C. B., Herrmann, F. R., ... \& Giannakopoulos, P. (2007). Morphological substrates of cognitive decline in nonagenarians and centenarians: a new paradigm? Journal of the Neurological Sciences, 257(1-2), 72-9.

Jones, K.T., Stephens, J.A., Alam, M., Bikson, M., Berryhill, M.E. (2015). Longitudinal Neurostimulation in Older Adults Improves Working Memory. PLoS ONE, 10(4). e0121904.

Kang, E.K., Baek, M.J., Kim, S. \& Paik, N.J. (2009). Non-invasive cortical stimulation improves post-stroke attention decline. Restorative Neurology and Neuroscience, $27(6), 645-650$.

Kim, S.H., Han, H.J., Ahn, H.M., Kim, S.A. \& Kim, S.E. (2012). Effects of five daily high-frequency rTMS on Stroop task performance in aging individuals. Neuroscience Research, 74(3-4), 256-260. 
Kraft, E. (2012). Cognitive function, physical activity, and aging: possible biological links and implications for multimodal interventions. Aging, Neuropsychology, and Cognition, 19(1-2), 248-263.

Kramer, J. H., Mungas, D., Reed, B. R., Wetzel, M. E., Burnett, M. M., Miller, B. L., ... \& Chui, H. C. (2007). Longitudinal MRI and cognitive change in healthy elderly. Neuropsychology, 21(4), 412-8.

Kubzansky, L.D., Berkman, L.F., Glass, T. \& Seeman, T.E. (1998). Is educational attainment associated with shared determinants of health in the elderly? Findings from the MacArthur Studies of Successful Aging. Psychosomatic Medicine, 60(5), $578-85$.

Lampit, A., Hallock, H. \& Valenzuela, M. (2014). Computerized cognitive training in cognitively healthy older adults: a systematic review and meta-analysis of effect modifiers. PLoS Medicine, 11, e1001756.

Di Lazzaro, V., Profice, P., Pilato, F., Capone, F., Ranieri, F., Pasqualetti, P., ... \& Dileone, M. (2010). Motor cortex plasticity predicts recovery in acute stroke. Cerebral Cortex, 20(7), 1523-1528.

Learmonth, G., Thut, G., Benwell, C.S.Y. \& Harvey, M. (2015). The implications of state-dependent tDCS effects in aging: Behavioural response is determined by baseline performance. Neuropsychologia 74, 108-119.

Lim, Y. Y., Pietrzak, R. H., Ellis, K. A., Jaeger, J., Harrington, K., Ashwood, T., ... \& Rowe, C. C. (2013). Rapid decline in episodic memory in healthy older adults with high amyloid- $\beta$. Journal of Alzheimer's Disease, 33(3), 675-679. 
Liuzzi, G., Freundlieb, N., Ridder, V., Hoppe, J., Heise, K., Zimerman, M., ... \& Hummel, F. C. (2010). The involvement of the left motor cortex in learning of a novel action word lexicon. Current Biology, 20(19), 1745-1751.

Lustig, C., Shah, P., Seidler, R. \& Reuter-Lorentz, P.A. (2009). Aging, Training and the Brain: A review and future directions. Neuropshycology Review, 19(4), 504-522.

McNab, F., Varrone, A., Farde, L., Jucaite, A., Bystritsky, P., Forssberg, H., \& Klingberg, T. (2009). Changes in cortical dopamine D1 receptor binding associated with cognitive training. Science, 323(5915), 800-802.

Mahon, B. Z., Kumar, N., \& Almeida, J. (2013). Spatial frequency tuning reveals interactions between the dorsal and ventral visual systems. Journal of Cognitive Neuroscience, 25(6), 862-871.

Manenti, R., Brambilla, M., Petesi, M., Ferrari, C. \& Cotelli, M. (2013). Enhancing verbal episodic memory in older and young subjects after non-invasive brain stimulation. Frontiers in Aging Neuroscience, 5(49), 1-9.

Mathers, C.D., Stevens, G.A., Boerma, T., White, R.A. \& Tobias, M.I. (2014). Causes of international increases in older age life expectancy. Lancet, 385(9967), 540-8.

Mattson, M.P., Maudsley, S. \& Martin, B. (2004). BDNF and 5-HT: A dynamic duo in age-related neuronal plasticity and neurodegenerative disorders. Trends in Neuroscience. 27(10), 589-594.

Meinzer, M., Lindenberg, R., Sieg, M.M., Nachtigall, L., Ulm, L. \& Flöel, A. (2014). Transcranial direct current stimulation of the primary motor cortex improves wordretrieval in older adults. Frontiers in Aging Neuroscience. 6(253), 1-9. 
Miniussi, C., Cappa, S. F., Cohen, L. G., Floel, A., Fregni, F., Nitsche, M. A., ... \& Walsh, V. (2008). Efficacy of repetitive transcranial magnetic stimulation/transcranial direct current stimulation in cognitive neurorehabilitation. Brain Stimulation, 1(4), 326-336.

Mungas, D., Harvey, D., Reed, B. R., Jagust, W. J., DeCarli, C., Beckett, L., ... \& Chui, H. C. (2005). Longitudinal volumetric MRI change and rate of cognitive decline. Neurology, 65(4), 565-71.

Nitsche, M. \& Paulus, W. (2009). Noninvasive brain stimulation protocols in the treatment of epilepsy: current state and perspectives. Neurotherapeutics, 6(2), 244250.

Nitsche, M. \& Paulus, W. (2011). Transcranial direct current stimulation - Update 2011. Restorative Neurology and Neuroscience, 29(6), 463-492.

Park, D.C. \& Reuter-Lorenz, P. (2009). The adaptive brain: aging and neurocognitive scaffolding. Annual Review of Psychology, 60, 173-96.

Park, S. H., Seo, J. H., Kim, Y. H., Ko, M. H. (2014). Long-term effects of transcranial direct current stimulation combined with computer-assisted cognitive training in healthy older adults. Neuroreport, 25(2), $122-6$.

Peters, R. (2006). Ageing and the brain. Postgraduate Medical Journal, 82(964), 84-88.

Poreisz, C., Boros, K., Antal, A. \& Paulus, W. (2007). Safety aspects of transcranial direct current stimulation concerning healthy subjects and patients. Brain Research Bulletin, 72(4-6), 208-214. 
Priori, A., Hallett, M. \& Rothwell, J.C. (2009). Repetitive transcranial magnetic stimulation or transcranial direct current stimulation? Brain Stimulation, 2(4), 241245.

Reis, J., Robertson, E. M., Krakauer, J. W., Rothwell, J., Marshall, L., Gerloff, C., ... \& Classen, J. (2008). Consensus: "Can tDCS and TMS enhance motor learning and memory formation?” Brain Stimulation, 1(4), 363.

Reis, J., Schambra, H. M., Cohen, L. G., Buch, E. R., Fritsch, B., Zarahn, E., ... \& Krakauer, J. W. (2009). Noninvasive cortical stimulation enhances motor skill acquisition over multiple days through an effect on consolidation. Proceedings of the National Academy of Sciences, 106(5), 1590-1595.

Ridding, M.C. \& Rothwell, J.C. (2007). Is there a future for therapeutic use of transcranial magnetic stimulation? Nature Reviews Neuroscience, 8(7), 559-567.

Romero, J.R., Ramirez, D.M., Aglio, L.S. \& Gugino, L.D. (2011). Brain mapping using transcranial magnetic stimulation. Neurosurgery Clinics of North America, 22(2), 141-52, vii.

Ross, L. a., McCoy, D., Coslett, H.B., Olson, I.R. \& Wolk, D. (2011). Improved proper name recall in aging after electrical stimulation of the anterior temporal lobes. Frontiers in Aging Neuroscience. 3(16), 1-8.

Rossi, S., Hallett, M., Rossini, P.M. \& Pascual-Leone, A. (2009). Safety, ethical considerations, and application guidelines for the use of transcranial magnetic stimulation in clinical practice and research. Clinical Neurophysiology, 120(12), 2008-39. 
Rusjan, P. M., Barr, M. S., Farzan, F., Arenovich, T., Maller, J. J., Fitzgerald, P. B., \& Daskalakis, Z. J. (2010). Optimal transcranial magnetic stimulation coil placement for targeting the dorsolateral prefrontal cortex using novel magnetic resonance image-guided neuronavigation. Human Brain Mapping, 31(11), 1643-1652.

Rutherford, G., Lithgow, B. \& Moussavi, Z. (2015). Short and Long-term Effects of rTMS Treatment on Alzheimer's Disease at Different Stages: A Pilot Study. Journal of Experimental Neuroscience, 9, 43-51.Salat, D.H. (2011). The Declining Infrastructure of the Aging Brain. Brain Connectivity, 1(4), 279-293.

Sandrini, M., Brambilla, M., Manenti, R., Rosini, S., Cohen, L.G. \& Cotelli, M. (2014). Noninvasive stimulation of prefrontal cortex strengthens existing episodic memories and reduces forgetting in the elderly. Frontiers in Aging Neuroscience, $6,289,1-9$.

Sandrini, M., Censor, N., Mishoe, J. \& Cohen, L.G. (2013). Causal role of prefrontal cortex in strengthening of episodic memories through reconsolidation. Current Biology, 23(21), 2181-4.

Sanes, J.N. \&Donoghue, J.P. (2000). Plasticity and primary motor cortex. Annual Review of Neuroscience, 23, 393-415.

Shafi, M.M., Westover, M.B., Fox, M.D. \& Pascual-Leone, A. (2012). Exploration and modulation of brain network interactions with noninvasive brain stimulation in combination with neuroimaging. European Journal of Neuroscience, 35(6), 805825. 
Siebner, H.R. \& Rothwell, J. (2003). Transcranial magnetic stimulation: New insights into representational cortical plasticity. Experimental Brain Research, 148(1), 116.

Siebner, H. R., Bergmann, T. O., Bestmann, S., Massimini, M., Johansen-Berg, H., Mochizuki, H., ... \& Pascual-Leone, A. (2009). Consensus paper: Combining transcranial stimulation with neuroimaging. Brain Stimulation, 2(2), 58-80.

Simis, M., Adeyemo, B.O., Medeiros, L.F., Miraval, F., Gagliardi, R.J. \& Fregni, F. (2013). Motor cortex-induced plasticity by noninvasive brain stimulation: a comparison between transcranial direct current stimulation and transcranial magnetic stimulation. Neuroreport, 24(17), 973-5.

Sparing, R. \& Mottaghy, F.M. (2008). Noninvasive brain stimulation with transcranial magnetic or direct current stimulation (TMS/tDCS)-From insights into human memory to therapy of its dysfunction. Methods, 44(4), 329-337.

Sullivan, G. M., \& Feinn, R. (2012). Using effect size-or why the P value is not enough. Journal of graduate medical education, 4(3), 279-282.

Wang, L., Zhang, Z., McArdle, J.J. \& Salthouse, T. (2008). Investigating Ceiling Effects in Longitudinal Data Analysis. Multivariate Behavioral Research, 43, 476-496.

Woods, A. J., Antal, A., Bikson, M., Boggio, P. S., Brunoni, A. R., Celnik, P., ... \& Knotkova, H. (2015). A technical guide to tDCS, and related non-invasive brain stimulation tools. Clinical Neurophysiology. 
Wong, S.-L., Wilczynski, N.L. \& Haynes, R.B. (2006). Comparison of top-performing search strategies for detecting clinically sound treatment studies and systematic reviews in MEDLINE and EMBASE. Journal of the Medical Library Association, 94(4), 451-455.

Zibly, Z., Shaw, A., Harnof, S., Sharma, M., Graves, C., Deogaonkar, M., \& Rezai, A. (2014). Modulation of mind: therapeutic neuromodulation for cognitive disability. Journal of Clinical Neuroscience, 21(9),1-5.

Zimerman, M. \& Hummel, F.C. (2010). Non-invasive brain stimulation: enhancing motor and cognitive functions in healthy old subjects. Frontiers in Aging Neuroscience, 2, 149. 


\section{Figure 2}

$\begin{array}{rrrrrrll}0,22 & 0,38 & 0,14 & -0,52 & 0,96 & 0,58 & 0,56 \text { Ross et al., 2011 (Right ATL) } \\ 0,61 & 0,39 & 0,15 & -0,14 & 1,37 & 1,59 & 0,11 \text { Ross et al., 2011 (Left ATL) } \\ 1,09 & 0,34 & 0,11 & 0,42 & 1,75 & 3,21 & 0,00 \text { Floel et al., 2012 } \\ 0,13 & 0,25 & 0,06 & -0,36 & 0,62 & 0,53 & 0,59 \text { Manenti et al., 2013 (Right DLPFC + PARC) } \\ 1,05 & 0,27 & 0,07 & 0,53 & 1,57 & 3,94 & 0,00 \text { Manenti et al., 2013 (Left DLPFC + PARC) } \\ 0,92 & 0,43 & 0,18 & 0,08 & 1,77 & 2,15 & 0,03 \text { Sandrini et al., 2014 (Anodal-NR) } \\ 0,85 & 0,43 & 0,18 & 0,01 & 1,69 & 1,99 & 0,05 \text { Sandrini et al., 2014 (Anodal-R) } \\ 0,74 & 0,41 & 0,16 & -0,05 & 1,54 & 1,83 & 0,07 \text { Cotelli et al., 2010 (Right DLPFC) } \\ 1,09 & 0,42 & 0,18 & 0,26 & 1,91 & 2,59 & 0,01 \text { Cotelli et al., 2010 (Left DLPFC) } \\ 0,73 & 0,34 & 0,12 & 0,05 & 1,40 & 2,11 & 0,03 \text { Meinzer et al., 2014 (Unilateral tDCS) } \\ 0,61 & 0,34 & 0,12 & -0,06 & 1,28 & 1,79 & 0,07 \text { Meinzer et al., 2014 (Bihemisferic tDCS) } \\ 0,18 & 0,32 & 0,10 & -0,45 & 0,80 & 0,55 & 0,58 \text { Fertonani et al., 2014 (Online tDCS) } \\ 0,00 & 0,32 & 0,10 & -0,62 & 0,62 & 0,00 & 1,00 \text { Fertonani et al., 2014 (Offline tDCS) } \\ 2,19 & 0,63 & 0,40 & 0,95 & 3,42 & 3,46 & 0,00 \text { Kim et al., 2012 } \\ 0,57 & 0,29 & 0,09 & -0,01 & 1,15 & 1,94 & 0,05 \text { Harty et al., 2014 } \\ 0,09 & 0,32 & 0,10 & -0,53 & 0,71 & 0,29 & 0,77 \text { Learmonth et al., 2014 (Right PPC) } \\ -0,11 & 0,32 & 0,10 & -0,73 & 0,51 & -0,34 & 0,73 \text { Learmonth et al., 2014 (Left PPC) } \\ 0,57 & 0,12 & 0,01 & 0,34 & 0,79 & 4,86 & 0,00 \text { Pooled effect size (random effects model) }\end{array}$

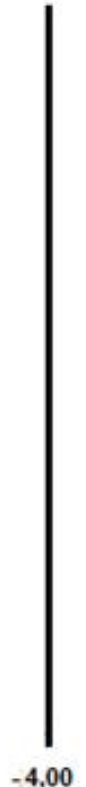

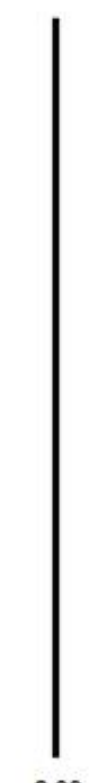

$-2,00$

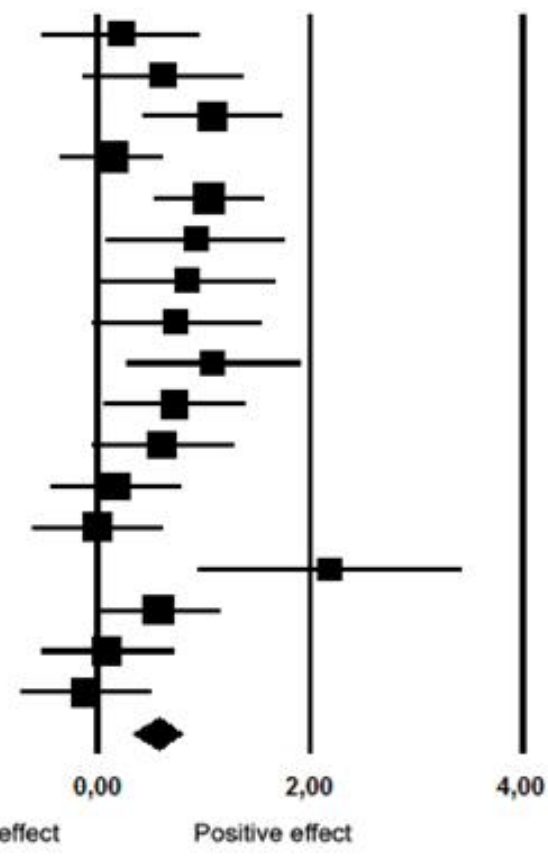

Relative weight

5,43

5,31

6,15

8,04

7,66

4,67

4,72

5,02

4.80

6,05

6,10

6,57

6,59

2,69

7,04

6,58

6,58

Figure 2 - Statistical summary and forest plot of effects sizes of the studies with available data for systematic comparison, with the pooled effect size. ATL - anterior temporal lobe; DLPFC - dorsolateral prefrontal cortex; PARC - parietal cortex; Anodal-NR - without reminder; Anodal-R - with reminder; tDCS - transcranial directr current stimulation; PPC - posterior parietal cortex. 
Figure 3

\section{Funnel Plot of Standard Error by Std diff in means}

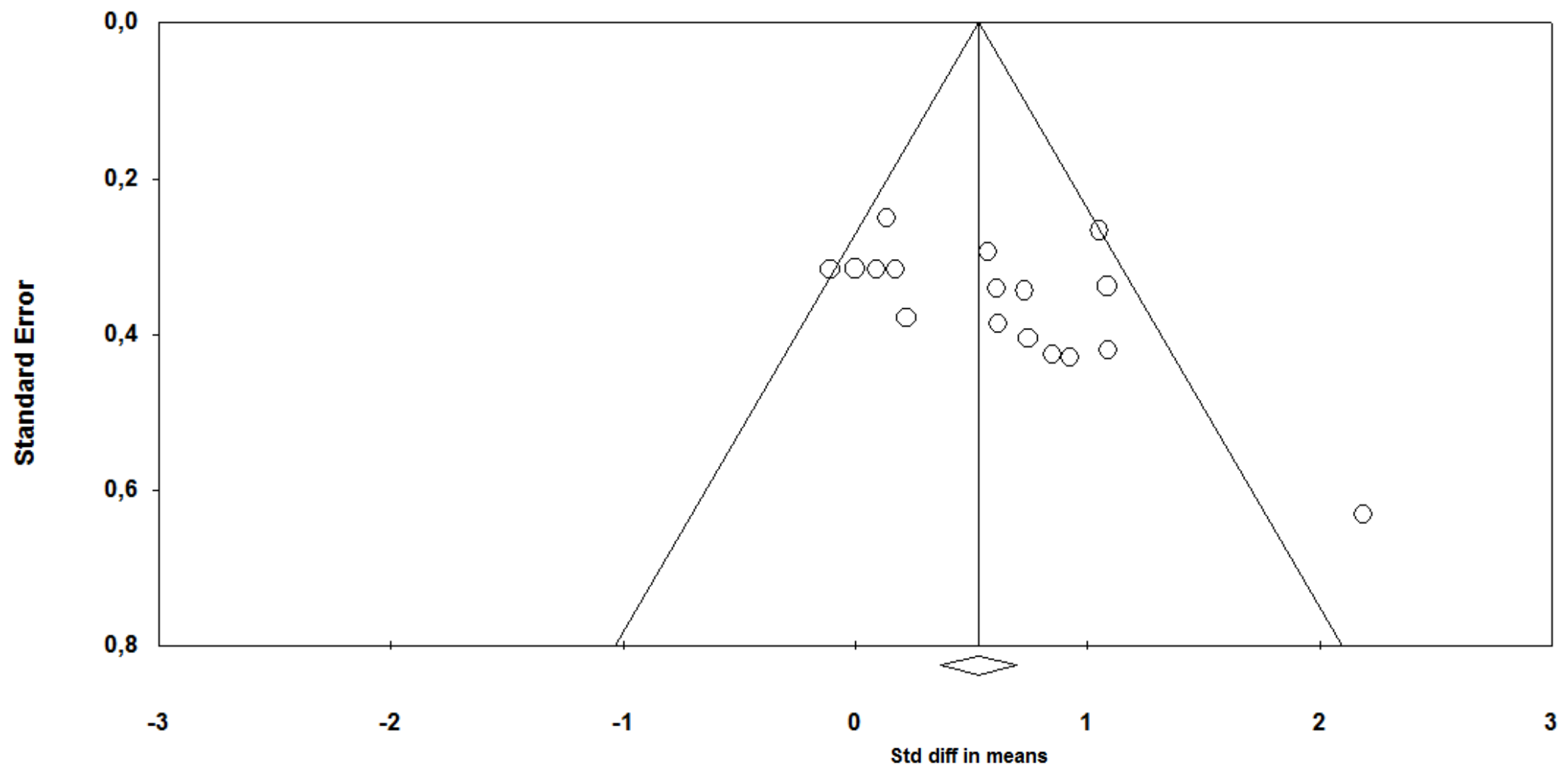

Figure 3 - Funnel plot (publication bias assessment) of the effect sizes (standardized mean differences) according to their standard errors. 
Table 1: Summary of the main features of included studies and report of adverse effects.

\begin{tabular}{|c|c|c|c|c|c|c|c|c|c|}
\hline Study & $n$ & Intervention & $\begin{array}{c}\text { Stimulation } \\
\text { site }\end{array}$ & $\begin{array}{c}\text { Stimulation } \\
\text { intensity/frequency }\end{array}$ & $\begin{array}{c}\text { Number of } \\
\text { sessions }\end{array}$ & Sham & $\begin{array}{l}\text { Cognitive domain } \\
\text { and task }\end{array}$ & Cognitive outcomes & $\begin{array}{c}\text { Adverse effects of } \\
\text { stimulation }\end{array}$ \\
\hline $\begin{array}{l}\text { Ross et al., } \\
2011\end{array}$ & $\begin{array}{l}14 \text { healthy older } \\
\text { adults }\end{array}$ & tDCS & $\begin{array}{l}\text { ATL (left or } \\
\text { right) }\end{array}$ & $1.5 \mathrm{~mA}$ & 3 & $\begin{array}{l}\text { Same montage; } \\
\text { current was } \\
\text { ramped up and } \\
\text { down for } 15 \\
\text { seconds }\end{array}$ & $\begin{array}{l}\text { Associative memory } \\
\text { Task: recall of faces } \\
\text { and places }\end{array}$ & $\begin{array}{c}\text { Anodal tDCS improved proper } \\
\text { naming of both famous faces and } \\
\text { landmarks with left and right ATL } \\
\text { stimulation, respectively. } \\
\text { The effects of face naming were of a } \\
\text { similar magnitude to that of young } \\
\text { adults, but only older adults } \\
\text { demonstrated an effect on place } \\
\text { naming. }\end{array}$ & $\begin{array}{c}\text { Not reported in the } \\
\text { manuscript. }\end{array}$ \\
\hline $\begin{array}{l}\text { Berryhill \& } \\
\text { Jones, } 2012\end{array}$ & $\begin{array}{c}25 \text { healthy older } \\
\text { adults }\end{array}$ & tDCS & $\begin{array}{l}\text { Prefrontal } \\
\text { cortex (left } \\
\text { or right) }\end{array}$ & $1.5 \mathrm{~mA}$ & 3 & $\begin{array}{l}\text { Same montage; } \\
\text { current was } \\
\text { ramped up and } \\
\text { down for } 20 \\
\text { seconds }\end{array}$ & $\begin{array}{l}\text { Working memory } \\
\text { Tasks: visual and } \\
\text { verbal 2-back } \\
\text { working memory } \\
\text { task }\end{array}$ & $\begin{array}{l}\text { tDCS was uniformly beneficial across } \\
\text { site and working memory task, but } \\
\text { only in older adults with more } \\
\text { education. In the less educated group, } \\
\text { tDCS provided no benefit to verbal or } \\
\text { visual working memory performance }\end{array}$ & $\begin{array}{c}\text { Not reported in the } \\
\text { manuscript }\end{array}$ \\
\hline
\end{tabular}




\begin{tabular}{|c|c|c|c|c|c|c|c|c|c|}
\hline $\begin{array}{l}\text { Flöel et al., } \\
2012\end{array}$ & $\begin{array}{c}20 \text { healthy older } \\
\text { adults }\end{array}$ & tDCS & TPC (right) & $1 \mathrm{~mA}$ & 2 & $\begin{array}{l}\text { Same montage: } \\
\text { current was } \\
\text { ramped up for } 30 \\
\text { seconds }\end{array}$ & $\begin{array}{l}\text { Episodic memory } \\
\text { Task: object- } \\
\text { location } \\
\text { learning/retrieval. }\end{array}$ & $\begin{array}{l}\text { Subjects performed comparably in the } \\
\text { learning task in the two conditions } \\
\text { (active and sham), but showed } \\
\text { improved recall one week after } \\
\text { learning with anodal tDCS compared } \\
\text { with learning with sham stimulation. }\end{array}$ & $\begin{array}{l}\text { Tingling sensation at } \\
\text { stimulation onset }\end{array}$ \\
\hline $\begin{array}{l}\text { Manenti et } \\
\text { al., } 2013\end{array}$ & $\begin{array}{c}\text { Experimental } \\
\text { group: } 32 \text { healthy } \\
\text { older adults } \\
\text { Control group: } 32 \\
\text { healthy young } \\
\text { volunteers }\end{array}$ & tDCS & $\begin{array}{c}\text { DLPFCs } \\
\text { and PARCs }\end{array}$ & $1.5 \mathrm{~mA}$ & 3 & $\begin{array}{l}\text { Same montage; } \\
\text { current was } \\
\text { ramped up and } \\
\text { down for } 10 \\
\text { seconds }\end{array}$ & $\begin{array}{l}\text { Episodic memory } \\
\text { Task: retrieval of } \\
\text { abstract and } \\
\text { concrete words } \\
\text { during the } \\
\text { application of either } \\
\text { active stimulation or } \\
\text { placebo. }\end{array}$ & $\begin{array}{l}\text { In young and in older subjects, anodal } \\
\text { tDCS applied during the retrieval } \\
\text { phase facilitates verbal episodic } \\
\text { memory. } \\
\text { tDCS applied over the left and right } \\
\text { regions DLPFC and PARC induced } \\
\text { better performance in young } \\
\text { participants; only tDCS applied over } \\
\text { the left regions increased retrieval in } \\
\text { older subjects }\end{array}$ & Itchiness and irritation \\
\hline
\end{tabular}




\begin{tabular}{|c|c|c|c|c|c|c|c|c|c|}
\hline $\begin{array}{l}\text { Sandrini et } \\
\text { al., } 2014\end{array}$ & $\begin{array}{c}36 \text { healthy older } \\
\text { adults }\end{array}$ & $\mathrm{tDCS}$ & $\begin{array}{l}\text { DLPFC } \\
\text { (left) }\end{array}$ & $1.5 \mathrm{~mA}$ & 4 & $\begin{array}{l}\text { Same montage; } \\
\text { current was } \\
\text { ramped up and } \\
\text { down for } 10 \\
\text { seconds }\end{array}$ & $\begin{array}{l}\text { Episodic memory } \\
\text { Task: learning } \\
\text { session, memory } \\
\text { reactivation and } \\
\text { memory retrieval }\end{array}$ & $\begin{array}{l}\text { Anodal tDCS over the left DLPFC } \\
\text { strengthened existing verbal episodic } \\
\text { memories and reduced forgetting } \\
\text { compared to sham stimulation. }\end{array}$ & Itchiness and irritation \\
\hline $\begin{array}{l}\text { Cotelli et } \\
\text { al., } 2010\end{array}$ & $\begin{array}{c}\text { Experiment 1: } 30 \\
\text { healthy older } \\
\text { adults } \\
\text { Experiment 2: } 13 \\
\text { healthy older } \\
\text { adults }\end{array}$ & rTMS & $\begin{array}{l}\text { DLPFC (left } \\
\text { and right) }\end{array}$ & $\begin{array}{l}90 \% \text { resting motor } \\
\text { threshold; } 20 \mathrm{~Hz}\end{array}$ & 1 & $\begin{array}{l}\text { A 3-cm-thick } \\
\text { piece of plywood } \\
\text { was applied to the } \\
\text { coil so that no } \\
\text { magnetic fields } \\
\text { reached the } \\
\text { cortex. }\end{array}$ & $\begin{array}{l}\text { Naming (Language) } \\
\text { Task: action and } \\
\text { object picture- } \\
\text { naming task }\end{array}$ & $\begin{array}{l}\text { The naming latency for actions was } \\
\text { shortened after active stimulation } \\
\text { compared to sham. Stimulation was } \\
\text { not observed to have any effect on } \\
\text { correctness of naming. }\end{array}$ & $\begin{array}{c}\text { None of the subjects } \\
\text { showed adverse } \\
\text { effects. }\end{array}$ \\
\hline $\begin{array}{l}\text { Meinzer et } \\
\text { al., } 2014\end{array}$ & $\begin{array}{c}18 \text { healthy older } \\
\text { adults }\end{array}$ & tDCS & M1 (left) & $1 \mathrm{~mA}$ & 3 & $\begin{array}{l}\text { Same montage; } \\
\text { current was } \\
\text { ramped up and } \\
\text { down for } 30 \\
\text { seconds }\end{array}$ & $\begin{array}{l}\text { Language } \\
\text { Task: semantic } \\
\text { word retrieval }\end{array}$ & $\begin{array}{l}\text { tDCS significantly improved word- } \\
\text { retrieval compared to sham-tDCS }\end{array}$ & $\begin{array}{c}\text { No adverse effects } \\
\text { were observed }\end{array}$ \\
\hline
\end{tabular}




\begin{tabular}{|c|c|c|c|c|c|c|c|c|c|}
\hline $\begin{array}{l}\text { Fertonani et } \\
\text { al., } 2014\end{array}$ & $\begin{array}{c}\text { Experimental } \\
\text { group: } \\
20 \text { healthy-aging } \\
\text { adults } \\
\text { Control group: } \\
20 \text { healthy young } \\
\text { subjects }\end{array}$ & tDCS & $\begin{array}{l}\text { DLPFC } \\
\text { (left) }\end{array}$ & $2 \mathrm{~mA}$ & 3 & $\begin{array}{l}\text { Same montage; } \\
\text { current was } \\
\text { ramped up and } \\
\text { down for } 10 \\
\text { seconds }\end{array}$ & $\begin{array}{l}\text { Naming (Language) } \\
\text { Task: picture- } \\
\text { naming }\end{array}$ & $\begin{array}{l}\text { Anodal stimulation of the left DLPFC } \\
\text { showed a facilitation effect on picture } \\
\text { naming in both young and healthy } \\
\text { aging adults. Anodal stimulation } \\
\text { applied to the left DLPFC during the } \\
\text { execution of a picture-naming task } \\
\text { modulated the behavioral } \\
\text { performance of healthy aging adults; } \\
\text { the offline tDCS application, which is } \\
\text { efficacious in young adults, did not } \\
\text { induce facilitation effects in older } \\
\text { adults. }\end{array}$ & $\begin{array}{c}\text { Pitching, itchiness and } \\
\text { burning }\end{array}$ \\
\hline
\end{tabular}




\begin{tabular}{|c|c|c|c|c|c|c|c|c|c|}
\hline $\begin{array}{l}\text { Kim et al., } \\
2012\end{array}$ & 16 healthy elderly & rTMS & $\begin{array}{l}\text { DLPFC } \\
\text { (left) }\end{array}$ & $\begin{array}{c}30 \% \text { of the maximal } \\
\text { stimulator output } \\
10 \mathrm{~Hz}\end{array}$ & 5 & $\begin{array}{l}\text { Coil was held at } \\
\text { an angle of } 90^{\circ} \text {, } \\
\text { and only one edge } \\
\text { of it rested on the } \\
\text { scalp. }\end{array}$ & $\begin{array}{l}\text { Inhibitory control } \\
\text { Task: Stroop task. }\end{array}$ & $\begin{array}{l}\text { Aging individuals showed facilitated } \\
\text { performance in the Stroop task when } \\
\text { it called for ignoring distracting } \\
\text { information. } \\
\text { Participants in the active HF-rTMS } \\
\text { stimulation group showed improved } \\
\text { performance in reaction time during } \\
\text { incongruent trials (i.e. those with } \\
\text { distracting information) after HF- } \\
\text { rTMS treatment compared with pre- } \\
\text { treatment trials. }\end{array}$ & $\begin{array}{l}\text { Not reported in the } \\
\text { manuscript }\end{array}$ \\
\hline $\begin{array}{l}\text { Harty et al., } \\
\qquad 2014\end{array}$ & $\begin{array}{c}106 \text { healthy older } \\
\text { adults }\end{array}$ & tDCS & $\begin{array}{l}\text { DLPFC } \\
\text { (left) }\end{array}$ & $1 \mathrm{~mA}$ & 6 & $\begin{array}{l}\text { Same montage; } \\
\text { current was } \\
\text { ramped up and } \\
\text { down for } 30 \\
\text { seconds }\end{array}$ & $\begin{array}{l}\text { Error awareness } \\
\text { Task: Go/ } \\
\text { No-go response } \\
\text { inhibition task }\end{array}$ & $\begin{array}{l}\text { Anodal tDCS over right DLPFC was } \\
\text { associated with a significant increase } \\
\text { in the proportion of performance } \\
\text { errors that were consciously detected. } \\
\text { No such improvements were observed } \\
\text { when the homologous contralateral } \\
\text { area was stimulated. }\end{array}$ & Itchiness \\
\hline
\end{tabular}




\begin{tabular}{|c|c|c|c|c|c|c|c|c|c|}
\hline $\begin{array}{l}\text { Learmonth } \\
\text { et al., } 2014\end{array}$ & $\begin{array}{c}\text { Experimental } \\
\text { group: } \\
20 \text { healthy older } \\
\text { adults } \\
\text { Control group: } \\
20 \text { healthy young } \\
\text { adults }\end{array}$ & $\mathrm{tDCS}$ & $\begin{array}{l}\text { Posterior } \\
\text { parietal } \\
\text { cortex } \\
\text { (left or } \\
\text { right) }\end{array}$ & $1 \mathrm{~mA}$ & 3 & $\begin{array}{l}\text { Same montage; } \\
\text { current was } \\
\text { ramped up and } \\
\text { down for } 30 \\
\text { seconds }\end{array}$ & $\begin{array}{l}\text { Spatial attention } \\
\text { Task: lateralized } \\
\text { visual detection task }\end{array}$ & $\begin{array}{l}\text { No differences were found in the } \\
\text { effects of stimulation between young } \\
\text { and older adults. Instead, the effects } \\
\text { of anodal tDCS were state-dependent } \\
\text { (i.e. related to task performance at } \\
\text { baseline / influenced by an } \\
\text { individual's baseline task } \\
\text { performance) whereas age did not } \\
\text { differentially affect response }\end{array}$ & $\begin{array}{l}\text { Tingling, itchiness, } \\
\text { burning sensation, } \\
\text { pain }\end{array}$ \\
\hline
\end{tabular}

tDCS - transcranial direct current stimulation; rTMS - repetitive transcranial magnetic stimulation; ATL - anterior temporal lobe; TPC -

temporoparietal cortex; DLPF - dorsolateral prefrontal cortex; and PARC - parietal cortex; M1 - primary motor cortex; HF-rTMS - high-

frequency repetitive transcranial magnetic stimulation. 
Table 2: Summary of Jadad individual assessments for each trial, based on the items: randomization, blinding, withdrawals and dropouts. Total Jadad scores refer to the score that is possible to obtain on the Jadad quality scale. Scores range from zero (low quality) up to a maximum of five points (high quality).

\begin{tabular}{|c|c|c|c|c|c|}
\hline & & & & & \\
\hline \multirow[b]{2}{*}{ Study } & \multirow[b]{2}{*}{ NIBS } & Randomization & Blinding & Withdrawals and dropouts & \multirow[b]{2}{*}{ Total } \\
\hline & & $\begin{array}{l}\text { (0) Randomization is not reported } \\
\text { (1) Study is reported as randomized, } \\
\text { but randomization is not fully appropriate } \\
\text { (2) Appropriate randomization is } \\
\text { reported. }\end{array}$ & $\begin{array}{l}\text { (0) Blinding is not reported } \\
\text { (1) Study is reported as blinded, but } \\
\text { blinding is not fully appropriate } \\
\text { (2) Appropriate blinding is reported. }\end{array}$ & $\begin{array}{l}\text { (0) The fate of participants in the trial is } \\
\text { unknown (withdrawals/dropouts not reported) } \\
\text { (1) The fate of all participants in the trial is } \\
\text { known (withdrawals/dropouts are described, } \\
\text { with reasons for exclusion) }\end{array}$ & \\
\hline Ross et al., 2011 & tDCS & 1 & 0 & 1 & 2 \\
\hline $\begin{array}{l}\text { Berryhill and Jones, } \\
2012\end{array}$ & $\mathrm{tDCS}$ & 0 & 0 & 0 & 0 \\
\hline Flöel et al., 2012 & $\mathrm{tDCS}$ & 1 & 2 & 1 & 4 \\
\hline Manenti et al., 2013 & tDCS & 1 & 1 & 0 & 2 \\
\hline Sandrini et al., 2014 & tDCS & 1 & 2 & 0 & 3 \\
\hline Cotelli et al., 2010 & rTMS & 0 & 0 & 0 & 0 \\
\hline
\end{tabular}




\begin{tabular}{|c|c|c|c|c|c|}
\hline Meinzer et al., 2014 & tDCS & 1 & 1 & 1 \\
\hline Fertonani et al., 2014 & tDCS & 0 & 1 & 0 \\
\hline Kim et al., 2012 & rTMS & 1 & 1 & 0 \\
\hline Harty et al., 2014 & tDCS & 1 & 1 & 1 \\
\hline Learmonth et al., 2015 & tDCS & 0 & 1 & 0 \\
\hline
\end{tabular}

